\title{
Rhinophyma - a clinical case and literature review
}

\author{
Stefan S. Konsulov, Taniel M. Minkov, lliya P. Yovchev, Denis A. Milkov, \\ Konstantina K. Piskova, S. Konsulov \\ Kaspela University Hospital, ENT Clinic, Department of ENT Diseases, \\ Medical University of Plovdiv, Bulgaria
}

\section{Introduction}

Rhinophyma is the most common clinical manifestation of phymatous rosacea. It is caused by a proliferation of the sebaceous glands and underlying connective tissue of the nose, which lead to disfiguring deformities. At an advanced stage, this condition can lead to psychological stress and an impaired respiratory function. The treatment is mainly surgical, but there is no ,gold standard“ for a therapeutic approach.

\section{Clinical case}

We present the case of a 69 year-old man who was admitted to the ENT Clinic at Kaspela University Hospital with a voluminous lobulated tumor formation on the outer nose, with a history of about 6 years. The examination revealed a hypertrophic thickening of the soft tissues of the nose, affecting and deforming the tip and two wings. The dilated skin pores, redness and prominent blood vessels characteristic of rhinophyma were visible (Fig. 1). The patient complained of psycho-emotional stress related to his appearance, and over the past few months, difficulty in nasal breathing. The patient did not report any other chronic illnesses, systemic treatments or allergies. Surgical excision under general anesthesia combined with bipolar electrocautery was used to remove the hypertrophic soft tissues and subsequently a free skin flap transplantation from the right thigh using a dermatome was preformed. No early or late postoperative complica- tions were reported. One year after the intervention, the patient reported an excellent cosmetic and functional effect of the performed surgical treatment.

\section{Discussion}

Rhinophyma is the most common clinical manifestation of phymatous rosacea. It is caused by a proliferation of the sebaceous glands and underlying connective tissue of the nose, which lead to disfiguring deformities. As early as the year 1490, one of Domenico Garlandaio's paintings, "An Old Man and His Grandson", depicts an elderly man with the typical appearance of an advanced rhinophyma. Later, in 1846, Ferdinand Ritter von Hebra first associated it with the final stage of development of rosacea, naming it "rhinophyma". In the past, rhinophyma has been mistakenly associated with excessive alcohol and caffeine consumption. This has contributed to the other names of the disease - "whiskey nose" and "rum nose." The name rhinophyma is derived from the Greek "rhis" meaning nose and "phyma" meaning skin tumor. Rhinophyma is the most common type of this skin tumor. Other localizations, and respectively names, include: forehead - metophyma; ear - otophyma; eyelids - blepharophyma; chin - gnatophyma.

The mechanisms of rosacea and rhinophyma formation are not fully understood, and various multifactorial circumstances are thought to be responsible, including a dysregulation of neurovascular structures and the innate immune system. Although 


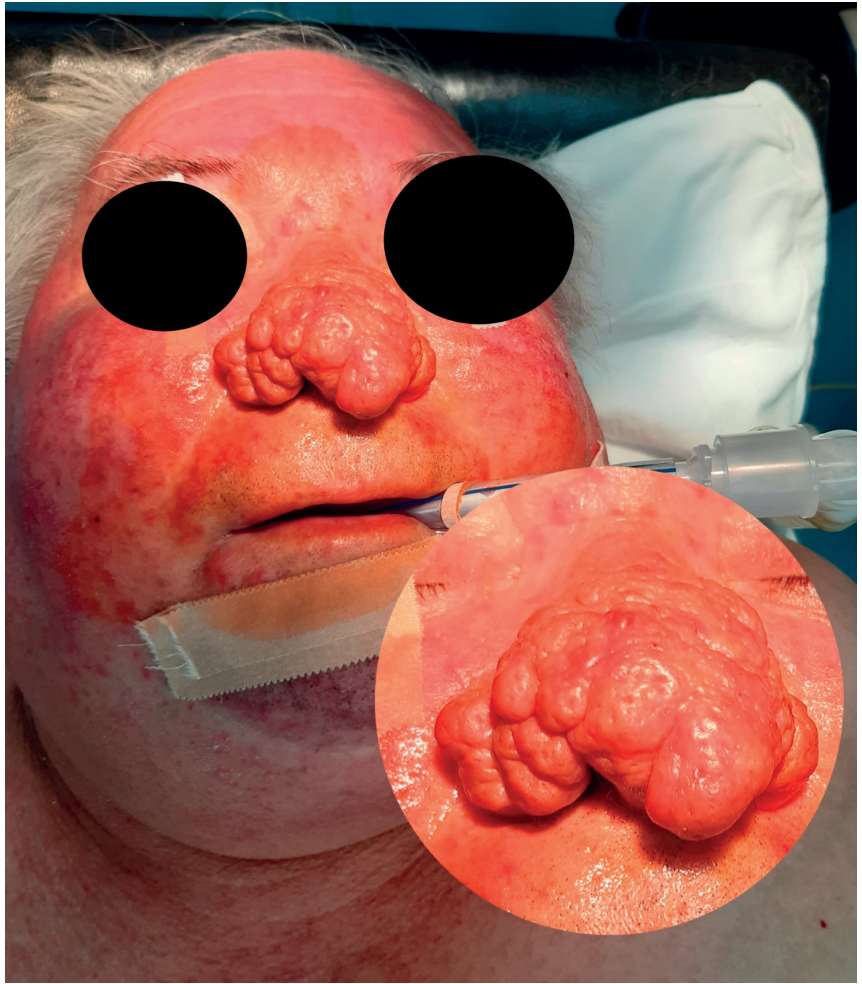

Figure 1. The characteristic of rhinophyma dilateted skin pores, reddening and prominant blood vessels - Third-degree rhinophyma

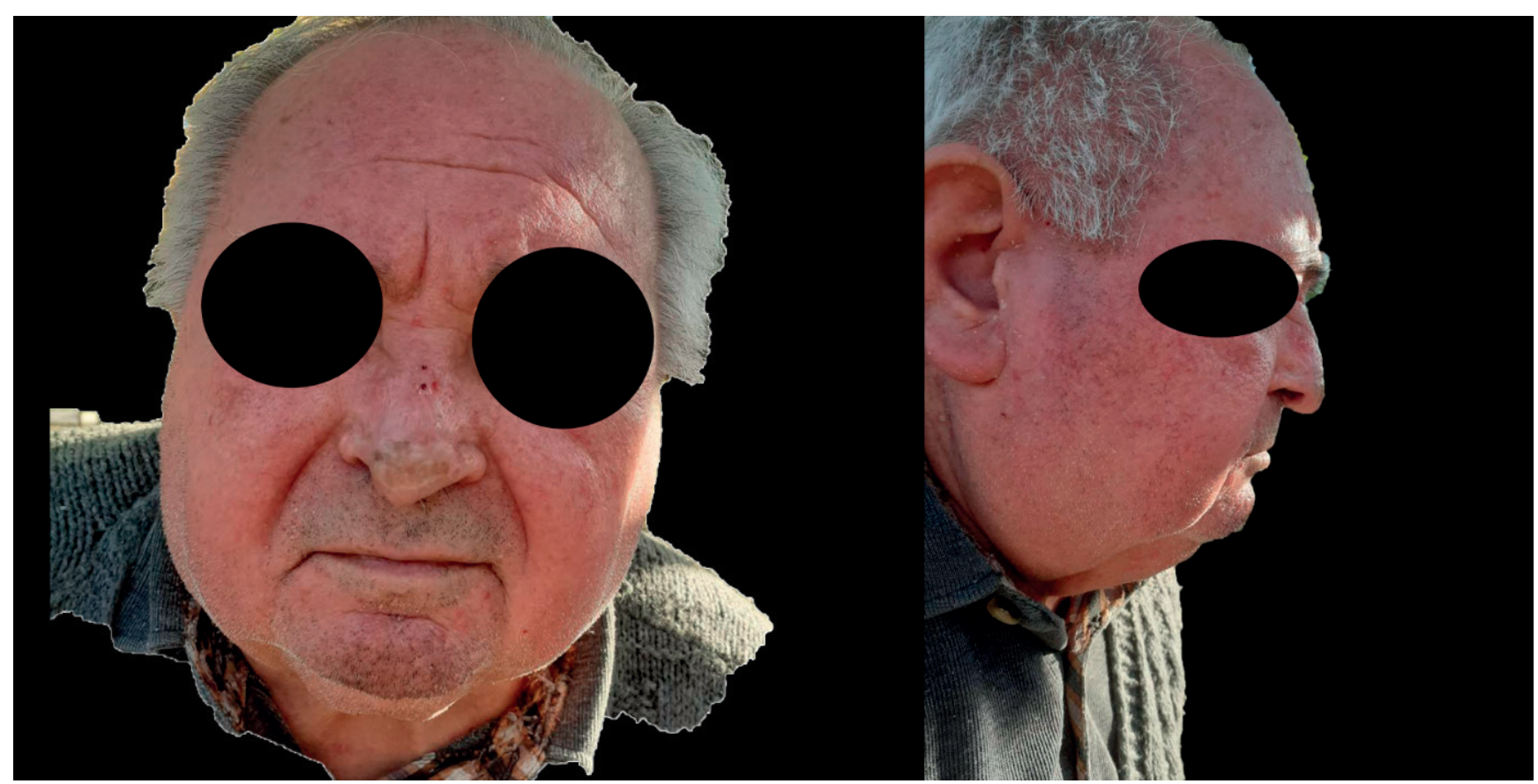

Figure 2. One year after surgical treatment

rosacea more often affects the female population, the development of rhinophyma is characteristic mainly of men, between 50-70 years of age, with a male to female ratio of 5-30: 1, assuming that the increased activity of androgens in men has an effect.

The diagnosis of rhinophyma is clinical and is characterized by an enlargement of the outer nose, thickening of the skin, dilatation of the skin pores and the appearance of telangiectasias. Additional imaging or laboratory tests are usually not needed, but a biopsy is required to confirm the diagnosis and to ruleout a malignancy.

Histologically, rhinophyma is manifested by a pro- 
liferation of the sebaceous glands and the underlying connective tissue of the nose, as well as with traces of a chronic inflammatory process. Different types of classifications exist, depending on the severity or subtypes of rhinophyma. The National Rosacea Society (NRS) offers a standard classification for rosacea and phymatous changes, according to their severity: 0 - Absent; $1-$ Follicles are patulous but no contour changes; 2 - contour change without nodularity; 3 - change in contour with the nodular component.

Despite their unique appearance, especially at an advanced stage, various diseases can mimic rhinophyma. These include basal cell carcinoma, squamous cell carcinoma, angiosarcoma, and other types of cancer. In addition, the simultaneous development of a malignant disease is possible. This makes the histological verification extremely important. In endemic areas with leishmaniasis, the typical enlargement of the nose may be a sign of the parasite. There are different approaches to treating rhinophyma, but there is currently no ,gold standard. “ Cases of x-ray treatment have been reported in the early 1920 s and 1930 s, but due to the increased risk of malignancy, radiotherapy is no longer recommended for the treatment of rhinophyma.

While topical medications are effective for rosacea, there is no evidence that this is the case with rhinophyma. According to some authors, oral doxycycline and isotretinoin can be used for an inflamed rhinophyma. There are studies showing the effectiveness of the drug tamoxifen, which is a non-steroidal anti-estrogen agent, which acts by lowering the fibroblast function and reducing the expression of the TGFb2 cytokine.

The advanced stages of rhinophyma require surgical treatment. The surgical methods are diverse and depend on the skills of the physician and the material base of the medical institution. The main surgical methods include surgical excision with or without a flap, cryosurgery, dermabrasion, laser surgery, coblation and others. The main goals in each type of treatment is to achieve thorough hemostasis and preservation of the cartilaginous skeleton of the nose, so as not to impair its respiratory function. The complications associated with the surgical techniques include scarring, prolonged recovery times, and impaired nasal respiratory function.

The postoperative care after most surgical techniques is standard and includes antibiotic ointments, creams with vitamins $\mathrm{A}$ and $\mathrm{D}$, and Vaseline, which help recovery. Some authors report the use of special dressings containing xeroform, calcium alginate, fibrin glue and others.

There are no large standardized studies on the longterm results after surgical treatment of rhinophyma. As the condition can lead to psychological stress and impaired respiratory function at an advanced stage, studies focusing on the impact on the quality of life based on psychological, aesthetic and functional outcomes are also needed.

\section{Conclusion}

Rhinofyma is a rare disease with an unclear pathophysiology, leading to a deformity of the outer nose, with subsequent impairment of respiratory function and psycho-emotional stress for the patient. The treatment is mainly surgical, but there is no ,gold standard." The described clinical case shows that the surgical approach used is a universal, affordable and effective method for achieving exceptional cosmetic and functional results in advanced rhinophyma.

\section{Bibliography:}

1. Dick MK, Patel BC. Rhinophyma. [Updated 2020 Aug 12]. In: StatPearls [Internet]. Treasure Island (FL): StatPearls Publishing; 2020 Jan-.

2. P. Clarós, M.-C. Sarr, F.-B. Nyada, A. Clarós, Rhinophyma: Our experience based on a series of 12 cases, European Annals of Otorhinolaryngology, Head and Neck Diseases, Volume 135, Issue 1, 2018, Pages 17-20

3. Chauhan R, Loewenstein SN, Hassanein AH. Rhinophyma: Prevalence, Severity, Impact and Management. Clin Cosmet Investig Dermatol. 2020;13:537-551

4. Chang, Daniel K. MD*; Savetsky, Ira L. MD †; Avashia, Yash J. MD†; Rohrich, Rod. J. MD*,† A Safe, Modern Treatment of Rhinophyma: The 5-Step Technique, Plastic and Reconstructive Surgery - Global Open: June 2020 - Volume 8 - Issue 6 - p e2620

5. Tambe SA, Nayak CS, Gala P, Zambare U, Nagargoje A. Management of Rhinophyma with Radio Frequency: Case Series of Three Patients. J Cutan Aesthet Surg. 2019;12(2):136-140. 